University of Wollongong

Research Online

Faculty of Engineering - Papers (Archive)

Faculty of Engineering and Information

Sciences

2007

\title{
Single electron effects and Bloch oscillations in high-TC superconductive tunneling junctions
}

Gustavo A. Alvarez

University of Wollongong, gustavo@uow.edu.au

X. L. Wang

University of Wollongong

SX. Dou

University of Wollongong, shi@uow.edu.au

M. Z. Wu

Colorado State University

Follow this and additional works at: https://ro.uow.edu.au/engpapers

Part of the Engineering Commons

https://ro.uow.edu.au/engpapers/453

\section{Recommended Citation}

Alvarez, Gustavo A.; Wang, X. L.; Dou, S X.; and Wu, M. Z.: Single electron effects and Bloch oscillations in high-TC superconductive tunneling junctions 2007.

https://ro.uow.edu.au/engpapers/453

Research Online is the open access institutional repository for the University of Wollongong. For further information contact the UOW Library: research-pubs@uow.edu.au 


\title{
Single electron effects and Bloch oscillations in high- $T_{C}$ superconductive tunneling junctions
}

\author{
G. A. Alvarez, ${ }^{\text {a) }}$ X. L. Wang, and S. X. Dou \\ Institute for Superconducting \& Electronic Materials, University of Wollongong, New South Wales 2522, \\ Australia \\ M. Z. Wu \\ Department of Physics, Colorado State University, Fort Collins, Colorado 80523
}

(Presented on 10 January 2007; received 31 October 2006; accepted 2 January 2007; published online 4 May 2007)

\begin{abstract}
We investigated the effects of single electron charging energy in high- $T_{C}$ superconductors. Various phenomena originated from Coulomb blockade were observable in $c$-axis $\mathrm{NdBa}_{2} \mathrm{Cu}_{3} \mathrm{O}_{7-\delta} / \mathrm{PrBa}_{2} \mathrm{Cu}_{3} \mathrm{O}_{7-\delta} / \mathrm{NdBa}_{2} \mathrm{Cu}_{3} \mathrm{O}_{7-\delta}$ superconducting tunnel junctions. The current-voltage characteristics show a Coulomb gap for Cooper pair tunneling when the charging energy exceeds the Josephson coupling energy. A crossover from Coulomb blockade of Cooper pair tunneling to a supercurrent is observed when the ratio of Josephson coupling energy to charging energy is increased. We found a regime in which aspects of the Coulomb blockade of tunneling coexist with features typical of Josephson tunneling. Under microwave irradiation of frequency $f$, the dynamic resistance $d V / d I$ as a function of the current $I$ showed sharp singularities at $I=2 n e f$, $n= \pm 1, \pm 2, \ldots$. This behavior appears to be very similar to the one predicted by the semiclassical theory of Bloch oscillations. (C) 2007 American Institute of Physics. [DOI: 10.1063/1.2712828]
\end{abstract}

The investigation of single electron tunneling (SET) effects has been limited so far to low- $T_{C}$ metal superconductors. The effects of charging energy in small Josephson junctions have been the subject of intensive theoretical study. ${ }^{1-3}$ Furthermore, experiments on very small junctions can provide important information about the validity of quantum mechanics on a microscopic scale. ${ }^{4-6}$

There are two energy scales in Josephson junctions. First, the Josephson coupling energy $E_{J}=h I_{J} / 4 \pi e$, which is the energy associated with the macroscopic variable $\phi$, that permits the transport of Cooper pairs between the electrodes ( $\phi$ is the phase difference between the junction electrodes, $I_{J}$ is the Josephson current, and $h$ is Planck's constant). Second, the charging energy $E_{C}=e^{2} / 2 C$, where $C$ is the junction capacitance, associated with $Q$ (the Cooper pair charge difference between the electrodes), that tends to localize the charge carriers. The behavior of the junction is determined by the ratio of these two energies. When $E_{J} \gg E_{C}$, the junction is in the conventional Josephson regime and the phase $\phi$ is well determined. When $E_{C} \gg E_{J}$, the phase is undetermined, so the system is subject to strong quantum fluctuations and the Coulomb blockade pins the Cooper pairs to the electrodes. The single electron tunneling processes, characterized by the tunneling conductance $1 / R_{T}$, should not be too strong $1 / R_{T} \leqslant 4 e^{2} / h$. The effect of strong quasiparticle tunneling between the electrodes modifies the energy levels and, above a critical strength, where a phase transition occurs, makes the single electron effects almost unobservable. ${ }^{3}$

$\mathrm{NdBa}_{2} \mathrm{Cu}_{3} \mathrm{O}_{7-\delta}(\mathrm{NBCO})$ thin films behave like superconducting two-dimensional (2D) systems. ${ }^{7}$ Superconducting to single electron tunneling transitions were observed in several

\footnotetext{
a) Author to whom correspondence should be addressed; electronic mail: gustavo@uow.edu.au
}

experiments in $c$-axis trilayer junctions fabricated from NBCO systems. The transitions occur in junctions where the charging energy $E_{C}$ is much larger than the thermal energy $k_{B} T$. They appear either as a Coulomb gap in the currentvoltage characteristics or as resonances if the frequency of an external radiation matches the average tunneling frequency of Cooper pairs.

The $\mathrm{NBCO} / \mathrm{PrBa}_{2} \mathrm{Cu}_{3} \mathrm{O}_{7-\delta}(\mathrm{PBCO}) / \mathrm{NBCO}$ trilayers used in this study were fabricated by pulsed laser deposition (PLD). Single film deposition is described elsewhere. ${ }^{7,8}$ The crystal structure of these trilayers was analyzed by using an $\mathrm{x}$-ray diffractometer (XRD) and a Rutherford backscattering spectrometer (RBS). Transmission electron microscopy (TEM) show quasihomoepitaxial growth between the NBCO/PBCO/NBCO interfaces. ${ }^{8}$ Planar junctions with widths varying from 5 to $10 \mu \mathrm{m}$ were fabricated from these $c$-axis NBCO/PBCO/NBCO trilayers by using standard photolithographic and ion milling techniques.

We measured the current-voltage characteristics (CVCs) of $\mathrm{NBCO} / \mathrm{PBCO} / \mathrm{NBCO}$ tunnel junctions with different $E_{C} / E_{J}$ ratios. By varying $E_{C} / E_{J}$, we have swept from the conventional Josephson effect regime $\left(E_{C} \ll E_{J}\right)$ well into the opposite regime, in which $E_{C} \gg E_{J}$, and the behavior is dictated by the charging energy $E_{C}$. This superconducting to single electron tunneling transition induced by the charging energy is shown in Fig. 1, where CVC are plotted for three samples with different $E_{C} / E_{J}$ ratios. The Josephson coupling energy $E_{J}$ is determined by the junction resistance $R_{N}$ and $\Delta$, the BCS gap of the superconducting electrodes: $E_{J}$ $=h I_{J} / 4 \pi e=\alpha \Delta / 2$, where $\alpha=R_{0} / R_{N}$ and $R_{0}=h / 4 e^{2}$ (where $R_{0}$ is the quantum resistance). For the estimation of the elementary charging energy $E_{C}=e^{2} / 2 C$, we use the junction capacitance value obtained from the asymptotic voltage offset $\Delta V$, $E_{C}=e \Delta V / 2 . \Delta V$ was determined by careful extrapolation of 


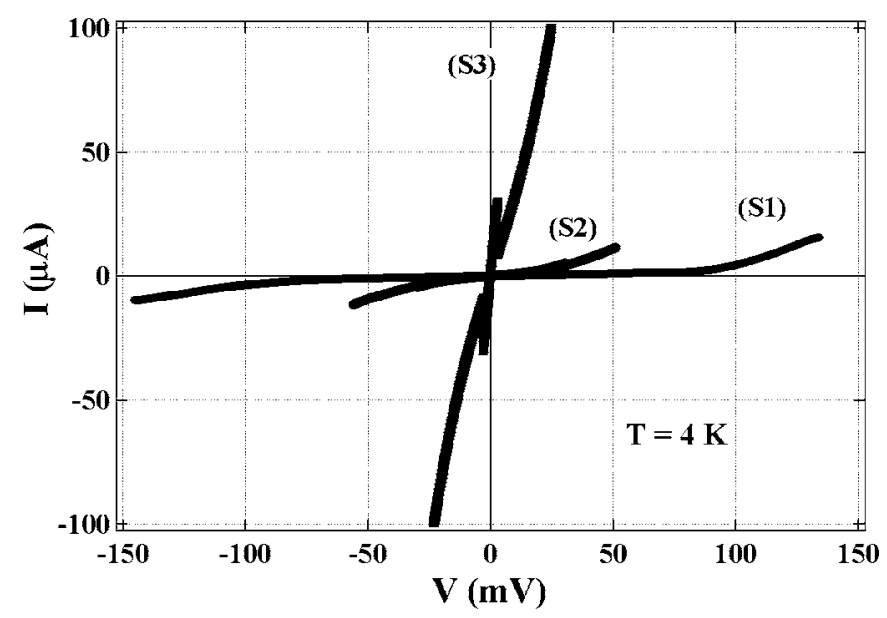

FIG. 1. (a) Current-voltage characteristics (CVCs) at $4.3 \mathrm{~K}$ for three $c$-axis $\mathrm{NBCO} / \mathrm{PBCO} / \mathrm{NBCO}$ trilayer junctions, (S1), (S2), and (S3), with different $E_{C} / E_{J}$ values given in Table I. With increasing $E_{C} / E_{J}$ ratio, the CVC changed from a supercurrent remnant to a sharp Coulomb blockade. (b) shows the enhanced center of (a).

the linear part of the $I-V$ curve from voltage greater than $2 \Delta / e$. All the parameters of the junctions are shown in Table I. These CVC responses are suggestive of the Coulomb blockade effect, observed in tunnel junctions, ${ }^{9-11}$ in which the charging energy is completely dominant. At low voltages, charge is trapped in the electrodes and the dynamic resistance is very large. For $V>e / 2 C$, however, the electron can acquire enough energy from the source to make tunneling energetically favorable.

The $I_{C}$ vs $T$ behavior of the high tunneling resistance $R_{T}$ samples is strikingly reentrant; $I_{C}$ decreasing with decreasing $T$ at intermediate temperatures and increasing again at low temperatures. We observed an anomalous decrease of the current as temperature is decreased below $40 \mathrm{~K}$ and then an increase at lower temperatures $(\sim 20 \mathrm{~K})$ (Fig. 2). This quasireentrant behavior may relate to the theories which consider the competition between thermal and quantum mechanical fluctuations and predict distinct reentrance. ${ }^{12,13}$ The effect of macroscopic quantum tunneling (MQT) on the system would be, indeed, to reduce the apparent critical current and introduce a fictitious noise temperature larger than the bath temperature of the junction. From microscopic theory, the current-biased Josephson junction can be represented as a quantum particle of mass $h^{2} / 8 E_{C}$, moving in a periodic tilted cosine potential- $E_{J} \cos \phi{ }^{14,15}$ Here $\phi$ is the phase difference across the junction. The zero voltage state of the junction corresponds to the confinement of the particle to one well of this potential. The necessary conditions for the observation of macroscopic quantum tunneling can be realized in the current-biased Josephson tunnel junction, where the

TABLE I. Calculated and measured parameters for $c$-axis NBCO/PBCO/ NBCO superconducting tunneling junctions.

\begin{tabular}{lcllll}
\hline \hline & $\alpha=R_{0} / R_{N}$ & $E_{C} / E_{J}$ & $C(\mathrm{ff})$ & $\mathrm{NBCO}(\mathrm{nm})$ & $\mathrm{PBCO}(\mathrm{nm})$ \\
\hline Sample 1 (S1) & 0.15 & 97 & 0.001 & $15 \pm 5$ & $10 \pm 2$ \\
Sample 2 (S2) & 0.7 & 4 & 0.005 & $20 \pm 5$ & $8 \pm 2$ \\
Sample 3 (S3) & 21.5 & 0.1 & 0.006 & $30 \pm 5$ & $5 \pm 2$ \\
\hline \hline
\end{tabular}

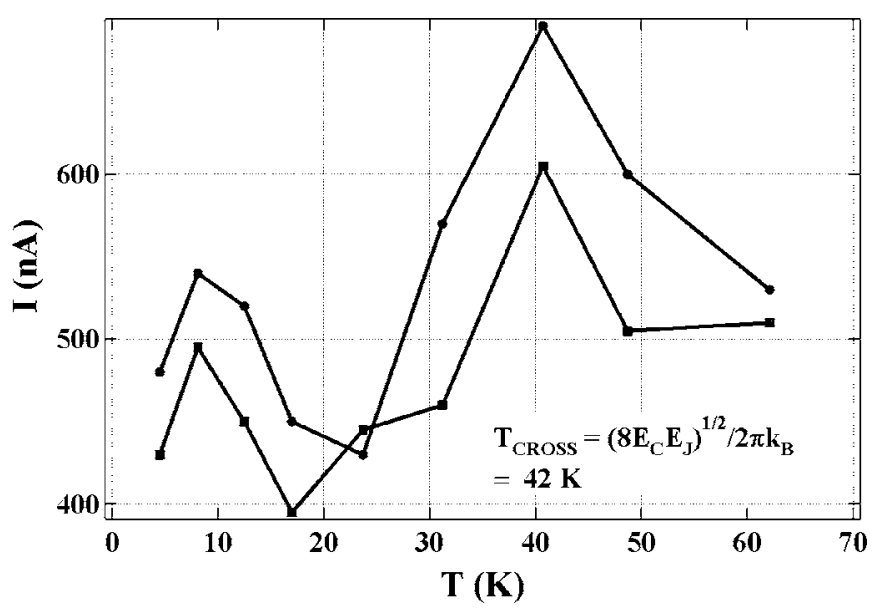

FIG. 2. Temperature dependence of critical current.

phase difference between the two superconductors is the macroscopic variable and the tunneling occurs from the zero voltage state to the nonzero voltage state.

The crossover temperature from thermal (temperature dependent, $k_{B} T \gg h \omega_{P} / 2 \pi$ ) to quantum (temperature independent, $\left.\quad k_{B} T \ll h \omega_{P} / 2 \pi\right)$ is predicted to be $T_{\text {cross }}$ $=\left(8 E_{C} E_{J}\right)^{1 / 2} / 2 \pi k_{B}$. Here, $\omega_{P}$ is the plasma frequency of small oscillations of the particle at the bottom of the well in the zerovoltage state. The temperature dependence of the critical current for two samples is shown in Fig. 2. The measured critical current $I_{C}$ is defined as the current found by extrapolation to $V=0$ from the $I-V$ curve at voltages below the gap voltage. These samples have a similar reentrant temperature dependence of the critical current, which has a maximum in the vicinity of $40 \mathrm{~K}$. For the junctions shown in Fig. 2, we calculated $T_{\text {cross }} \sim 42 \mathrm{~K}$, which is very close to the experimental value $(40 \mathrm{~K})$. These results imply that the effects of MQT play an important role in our junctions.

With increasing ratio $E_{C} / E_{J}$, the quantum mechanical behavior of the junction should become more noticeable. For high $E_{C} / E_{J}$, the behavior of the junction should be governed by a band energy spectrum. External currents cause a sweep of the junction Bloch state through this band spectrum. Bloch oscillations with the frequency to current relation $f_{B}$ $=I / 2 e$ were predicted $^{1}$ to take place in small Josephson junctions due to correlated tunneling of Cooper pairs. This is one of the most important phenomena arising from Coulomb blockade. The Bloch oscillations should be observed by applying an ac drive of frequency $f$ to the junction. In this case a complete phase locking of the oscillations by harmonics of the ac drive would result in voltage steps in the $I-V$ characteristics of the junction, located at quantized levels of the current $^{1}$

$$
I=2 \text { nef }, \quad n= \pm 1, \pm 2, \ldots .
$$

Experimental observations of Bloch oscillations in low- $T_{C}$ superconductors have been reported ${ }^{17}$ and explained within the context of the "orthodox" (semiclassical) theory of Bloch oscillations. ${ }^{1}$ In real experiments, the phase locking due to nonvanishing thermal fluctuations and single electron tunneling ${ }^{9,17}$ is far from the ideal situation giving nonhorizontal voltage steps and broadening the spectrum. However, 

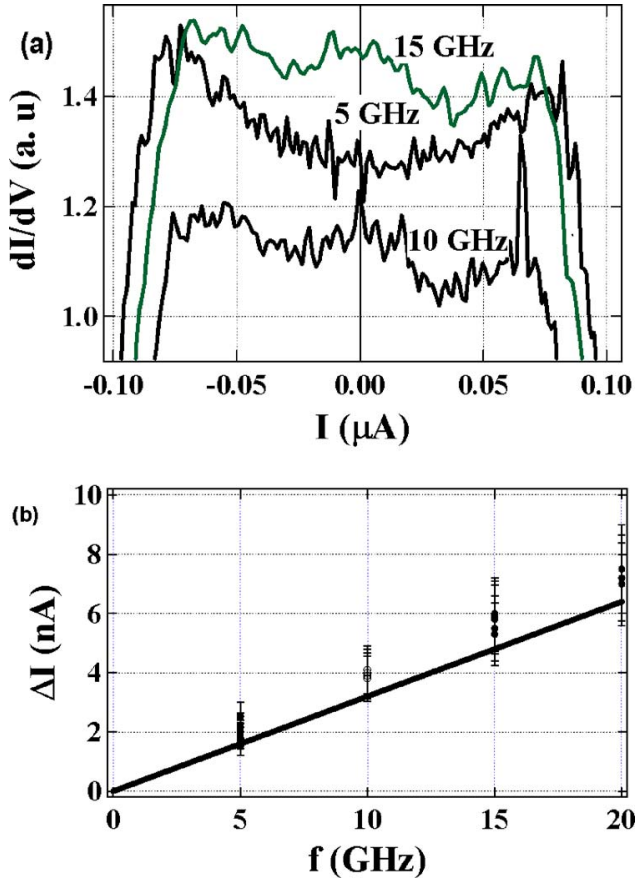

FIG. 3. (a) Measurement of the dynamic resistance $d V / d I$ vs the current $I$ for several values of $f$ at certain power levels. The arrows mark the maxima of the microwave-induced singularities. (b) The current $\Delta I$, which is the distance between the microwave-induced singularities of $d V / d I$, plotted as a function of the applied frequency $f$. The points show experimental data, while the line corresponds to the theoretical prediction $I=2 e f$.

one can expect to observe more or less sharp singularities atthe current values given by Eq. (1). By irradiating the junctions with a rf signal $(f=2-20 \mathrm{GHz})$, we could induce steplike features in the $I-V$ curves. These steps were not very sharp in the $I-V$ curves, but appear as oscillations in the $d V / d I$ spectrum. Figure 3(a) shows data from one of the samples. The oscillation amplitude changed with frequency. Within the experimental accuracy, the oscillation amplitude was found to follow the relation $\Delta I=2 n e f, n= \pm 1, \pm 2, \ldots$, as shown in Fig. 3(b). The error bars of Fig. 3(b) give our experimental accuracy in determining the oscillation amplitude. Within this accuracy, the points are relatively close to the theoretical line $I= \pm 2 n e f$. We rule out the Shapiro steps as being responsible for the rf induced features in $d V / d I$. The voltage scale of these features is much larger than the expected value for the Shapiro steps. For example, under $5 \mathrm{GHz}$ irradiation, the $d V / d I$ curve shows oscillations with amplitude of $V= \pm 2 \mathrm{mV}$, instead of $V= \pm 2 \mu \mathrm{V}$ as expected for Josephson oscillations. In conclusion, we believe that the observed microwave-induced singularities at the current values of $I= \pm 2 n e f$ give convincing evidence of the existence of continuous wave Bloch oscillations in our junctions.

The observed features find an explanation within the framework of the theory of correlated tunneling of Cooper pairs. We believe that the observed superconducting to single electron tunneling transitions were induced by the localization of charge carriers and the corresponding enhancement of quantum mechanical phase fluctuations of the superconducting order parameter. A quantitative comparison in order to measure the relevant parameters of the system independently is, however, hindered by the absence of quantitative theoret- ical predictions for high- $T_{C}$ superconductors, a significant uncertainty in important parameters, and by experimental restrictions.

The physical nature of the tunnel structure plays a crucial role in determining their electric characteristics. High quality NBCO/PBCO layers grow quasihomoepitaxially and form structures without an interface layer at unit-cell level (the unit cell $\sim 1.12 \mathrm{~nm}$ ). ${ }^{8}$ We believe that the charges are distributed inhomogeneously in the electrodes, accumulating in that thin layer next to the PBCO barrier. Since the charge density is the result of a displacement of the electronic charge with respect to the ionic background, the integrated density can take any continuous value. Only the charges next to the barrier interact. Their interaction can be described by the capacitance $C$ between the NBCO electrodes.

By making the assumption that the NBCO/PBCO interface layer is embedded in a "uniform" $\mathrm{PBCO}$ dielectric medium of a constant $\varepsilon(\sim 40)$, this implies that the voltage spacing $\Delta V=e / C$, here $C$ can be approximated by $C$ $\approx 2 \pi \varepsilon_{0} \varepsilon d$, where $d$ is the PBCO thickness and $\varepsilon_{0}=8.8542$ $\times 10^{-12} \mathrm{~F} / \mathrm{m}$. Since the uniform PBCO dielectric medium in which the NBCO/PBCO interface is embedded has a constant $\varepsilon=40$, then $C$ will be of the order of $2.2 \times 10^{-17} \mathrm{~F}$ for a PBCO thickness of $10 \mathrm{~nm}$, which is consistent with the values we obtained from our experimental data.

Finally, these high $E_{C} / E_{J}$ ratio junctions prominently exhibit charging effects and quantum coherence. We consider this as the clearest observation so far of macroscopic quantum coherent effects for single junctions in high- $T_{C}$ superconductors.

We are indebted to Professor C. Cook for his advice and support. We thank Dr. T. Puzzer, Dr. J. Horvat, and Dr. T. Silver for valuable discussions. This work was partially supported by the Australian Research Council and a start-up start grant from the University of Wollongong.

${ }^{1}$ K. K. Likharev and A. B. Zorin, J. Low Temp. Phys. 59, 347 (1985). ${ }^{2}$ F. Guinea and G. Schön, J. Low Temp. Phys. 69, 219 (1987); A. Furusaki and M. Ueda, Phys. Rev. B 45, 10576 (1992).

${ }^{3}$ U. Geigenmüller and G. Schön, Physica B 152, 186 (1988).

${ }^{4}$ A. I. Larkin, K. K. Likharev, and Yu. N. Ovchinnikov, Physica B \& C 126B, 414 (1984).

${ }^{5}$ H. S. J. van der Zant, W. J. Elion, L. J. Geerligs, and J. E. Mooij, Phys. Rev. B 54, 10081 (1996), and references therein.

${ }^{6}$ P. Delsing, C. D. Chen, D. B. Haviland, Y. Harada, and T. Claeson, Phys. Rev. B 50, 3959 (1994), and references therein.

${ }^{7}$ G. A. Alvarez, K. Hayashi, and Y. Enomoto, in Advances in Superconductivity VIII, Proceedings of the Eighth International Symposium on Superconductivity (ISS '95), 30 October-2 November 1995, Hamamatsu, Japan (Springer-Verlag, Tokyo, 1996), p. 1055.

${ }^{8}$ G. A. Alvarez, J. Wen, F. Wang, and Y. Enomoto, Jpn. J. Appl. Phys., Part 2 35, L1050 (1996).

${ }^{9}$ D. V. Averin and K. K. Likharev, J. Low Temp. Phys. 62, 345 (1986).

${ }^{10}$ K. Mullen, E. Ben-Jacob, and Z. Schuss, Phys. Rev. Lett. 60, 1097 (1988).

${ }^{11}$ L. J. Geerligs, V. F. Anderegg, J. Romijn, and J. E. Mooij, Phys. Rev. Lett. 65, 377 (1990).

${ }^{12}$ M. Iansiti, A. T. Johnson, W. F. Smith, H. Rogalla, C. J. Lobb, and M. Tinkham, Phys. Rev. Lett. 59, 489 (1987).

${ }^{13}$ M. H. Devoret et al., Phys. Rev. Lett. 55, 1908 (1985).

${ }^{14}$ W. C. Stewart, Appl. Phys. Lett. 12, 277 (1968); D. E. MacCumber, J. Appl. Phys. 39, 3133 (1968).

${ }^{15}$ V. Ambegaokar et al., Phys. Rev. Lett. 48, 1745 (1982); U. Eckern, V. Ambegaokar, and G. Schön, Phys. Rev. B 30, 6419 (1984).

${ }^{16}$ I. Affleck, Phys. Rev. Lett. 46, 388 (1981).

${ }^{17}$ L. S. Kuzmin and D. B. Haviland, Appl. Phys. Lett. 67, 2890 (1991). 